\title{
Research
}

Chris C Butler, Meredith KD Hawking, Anna Quigley and Cliodna AM McNulty

\section{Incidence, severity, help seeking, and management of uncomplicated urinary tract infection:}

\author{
a population-based survey
}

\begin{abstract}
\section{Background}

Limited knowledge of the population incidence and management of uncomplicated urinary tract infection (UTI) limits information provision and interventions to enhance care in the community.

\section{Aim}

To describe incidence and severity, help seeking, and management of UTI from a population perspective.

\section{Household survey in England in 2014}

Design and setting

\section{Method}

In total, a random sample of 2424 females aged $\geq 16$ years were interviewed in their own homes using computer-assisted interviewing about their UTI symptoms, help seeking, and management. Data were weighted by sex, age, ethnicity, working status, social grade, and housing tenure, and Government Office Region to be broadly representative of the general population.

\section{Results}

Of the females interviewed, 892 (37\%) reported having had at least one UTI in their lifetime (29\% had more than one episode). In the past year, $11 \%$ of all females reported a UTI and $3 \%$ recurrent UTI ( $\geq 3$ or more). Of those who had ever had a UTI, 48\% rated their last UTI as fairly or very severe. In total, 95\% consulted a health professional; $65 \%$ at their local GP practice during routine consulting hours. Out-of-hours consulting was uncommon but more prevalent in younger females. Of those contacting a health professional, $76 \%$ had a urine test, $74 \%$ were prescribed an antibiotic, but only $63 \%$ of these

INTRODUCTION

Symptoms associated with urinary tract infection (UTI) are one of the commonest acute reasons for adult females to seek health care, and are often severe. ${ }^{1-4}$ UTI accounts for an important proportion of antibiotics prescribed in primary care. ${ }^{5}$ The overall costs associated with UTI in the US have been estimated at $\$ 2$ billion per annum. ${ }^{6}$ Although studies of urine culture findings have been done in hospital outpatient clinics, ${ }^{7}$ important questions remain about incidence, help seeking, and the investigation and management of this condition in the community. Widespread variation in the primary care management of the condition suggests more evidence is needed to optimise standardised care..$^{8,9}$ There may be a 'gap' between clinicians' perceptions of the severity of their patients' symptoms and the perceptions of patients themselves, but knowledge of the symptom burden experienced by patients comes largely from clinical trials, which may not reflect the majority of episodes in the community or during routine primary care..$^{10} \mathrm{~A}$ better understanding of patients perspectives could inform interventions to optimise help seeking and management of UTI. Therefore a community-based interview survey was conducted on the incidence,
\end{abstract} reported taking the antibiotic. Delayed antibiotic prescribing was rare.

\section{Conclusion}

UTI symptoms are common; most females consult in general practice, and are prescribed antibiotics, but one-third report not taking the antibiotics as prescribed. Benefit and harms in those taking, and not taking, antibiotics need to be better understood in order to improve help seeking, management, and adherence. Urine tests and antibiotics could be reduced by basing empirical antibiotics on symptoms, and increasing use of back-up prescriptions.

\section{Keywords}

anti-infective agents; consulting; diagnostic test, routine; epidemiology; primary care; urinary tract infection.
CC Butler, FRCGP, professor of primary care Nuffield Department of Primary Care Health Cwm Taf University Health Board, Wales. MKD Hawking, MPH, research assistant, Public Health England Primary Care Unit, Microbiology Department, Gloucestershire Royal Hospital, Gloucester, UK. A Quigley, head of health research, Ipsos MORI Social Research Institute, London, UK. CAM McNulty, FRCPathol, head of unit, Public Health England Primary Care Unit, and visiting honorary professor, Cardiff University, Microbiology Department, Gloucestershire Royal Hospital, Gloucester, UK. Sciences, University of Oxford, Oxford, and GP. symptom burden, help seeking, and selfand primary care management of UTI.

\section{METHOD}

Ipsos MORI lpart of the Ipsos Group and a leading UK research company with global reach) interviewed in 2014 a random sample of 2424 females aged $\geq 16$ years in England in their own homes using computer-assisted interviewing. Fieldwork was carried out in three waves of the Ipsos MORI omnibus between 28 February and 20 March 2014. The Face-to-Face Omnibus used a controlled form of random location sampling (known as 'random locale'). Random locale is a dual-stage sample design, taking as its universe Sample Units, a bespoke amalgamation of Output Areas IOAs: the basic building block used for output from the Census) in Great Britain. These OAs are regrouped into Sample Units taking account of their ACORN characteristics. A total of 170-180 PSUs (Primary Sampling Units) are randomly selected from the stratified groupings with probability of selection proportional to size.

At the second stage, usually two adjacent OAs, made up of approximately 125 addresses each, are randomly selected from each PSU; this then becomes the secondary sampling unit.

\section{Address for correspondence}

Chris C Butler, Professor of Primary Care, Nuffield Department of Primary Care Health Sciences, Medical Sciences Division, University of Oxford, New Radcliffe House, Radcliffe Observatory Quarter, Woodstock Road, Oxford, OX2 6NW.

E-mail: christopher.butleraphc.ox.ac.uk Submitted: 26 March 2015; Editor's response: 30 April 2015; final acceptance: 27 May 2015. CBritish Journal of General Practice

This is the full-length article (published online 28 Sep 2015) of an abridged version published in print. Cite this article as: $\mathbf{B r} \mathbf{J}$ Gen Pract 2015 DOI: 10.3399/bjgp15X686965 


\section{How this fits in?}

Symptoms attributable to uncomplicated urinary tract infection (UTI) are common and account for considerable personal suffering and healthcare resource use. However, UTI incidence in the general population, help seeking, and management in primary care are not well described. Our populationbased survey found that UTI symptoms are common, most females consult in general practice when they occur, and are prescribed antibiotics, but that onethird report not taking the antibiotics as prescribed. High levels of testing and prescribing might be reduced by increased use of symptom-based prescribing and use of back-up antibiotics.

Interviewers were given age and sex quotas of respondents for each OA. Interviewers went door-to-door and invited females over 16 years who answered to participate. If that person refused, interviewers could invite another member of the household. Households were visited throughout the week during the day, evening, and weekend so working people could be included. Interviewers did not revisit non-responding households. About one interview was completed for approximately every three or four doors on which interviewers knocked.

Data were weighted by sex, age, ethnicity, working status, social grade, housing tenure, and Government Office Region (GOR) so that they are broadly representative

\section{Box 1. UTI questions administered using computer-assisted personal interviewing}

- Have you ever had a UTI?

- Approximately how many UTIs have you had in your lifetime?

- Approximately when was your last UTI?

- Approximately how many UTIs have you had in the last year?

- To what extent, if at all, did your most recent UTI affect your daily life?

- Thinking about your most recent UTI, how severe, if at all, were your symptoms?

-Which, if any, of the following healthcare professionals did you contact as a result of your most recent UTI?

- You said you visited a healthcare professional. Why did you do this?

- Did you have a urine test?

-Which, if any, of the following [options about getting the results] apply to you?

- Were you prescribed antibiotics?

- How were the antibiotics prescribed to you?

- What other advice or treatment, if any, were you given?

-Which of the following actions, if any, did you take as a result of your most recent UTI? of the population. Base sizes of less than 100 should be treated with caution and these results seen as indicative only. Bases of less than 30 are presented as numbers rather than percentages; these should be treated with extreme caution and seen as indicative only.

The interview schedule was developed collaboratively by the authors (Box 1), and with input from the Ipsos MORI health team.

The survey provided the following description of a UTI: 'Urinary tract infections (UTIs) are often called urine, water or bladder infections or cystitis. They can give pain when passing urine and a need to pass urine more often.'

\section{RESULTS}

In total, 2424 females were surveyed and, unless otherwise stated, results are based on those respondents who reported ever having had a UTI in their lifetime $(n=892)$.

\section{Incidence and severity}

Over a third $(37 \%, 892 / 2424,95 \% \mathrm{Cl}=35.1 \%$ to $38.9 \%$ ) of all the females reported having had at least one UTI in their lifetime (Figure 1). This varied by age group (16-34 years, 36\%; 35-54 years, 42\%; and $55+$ years, $33 \%)$ and social class (42\% of social grade ABC1 compared with 30\% of C2DE). Of these, $79 \%$ had experienced more than one episode in their lifetime, and $57 \%$ reported having had three or more UTI episodes. Eleven per cent of all females surveyed reported a UTI in the last year $(95 \% \mathrm{Cl}=9.8 \%$ to $12.2 \%)$, with $6 \%(95 \% \mathrm{Cl}=5.1 \%$ to $6.9 \%)$ of all females reporting more than one episode during the past year. Three per cent $195 \% \mathrm{Cl}=2.3 \%$ to $3.7 \%$ ) of the female population surveyed reported experiencing recurrent UTI (three or more episodes in the past year).

Of 892 females who had ever had a UTI rated, $13 \%(95 \% \mathrm{Cl}=10.8 \%$ to $15.2 \%)$ deemed the symptoms of their last UTI as very severe (by choosing 9 or 10 on a scale of 0 to 10 ), whereas $35 \%$ rated their symptoms as fairly severe (by choosing 6-8 on a scale of 0-10). Thirty-seven per cent reported their most recent UTI affected their daily life a fair amount and 15\% a great deal when asked, 'To what extent, if at all, did your most recent UTI affect your daily life?'

\section{Help seeking}

The vast majority $195 \% ; 95 \% \mathrm{Cl}=93.6 \%$ to 96.4\%) of females who had ever had a UTI reported contacting a health professional about their most recent attack; severe 


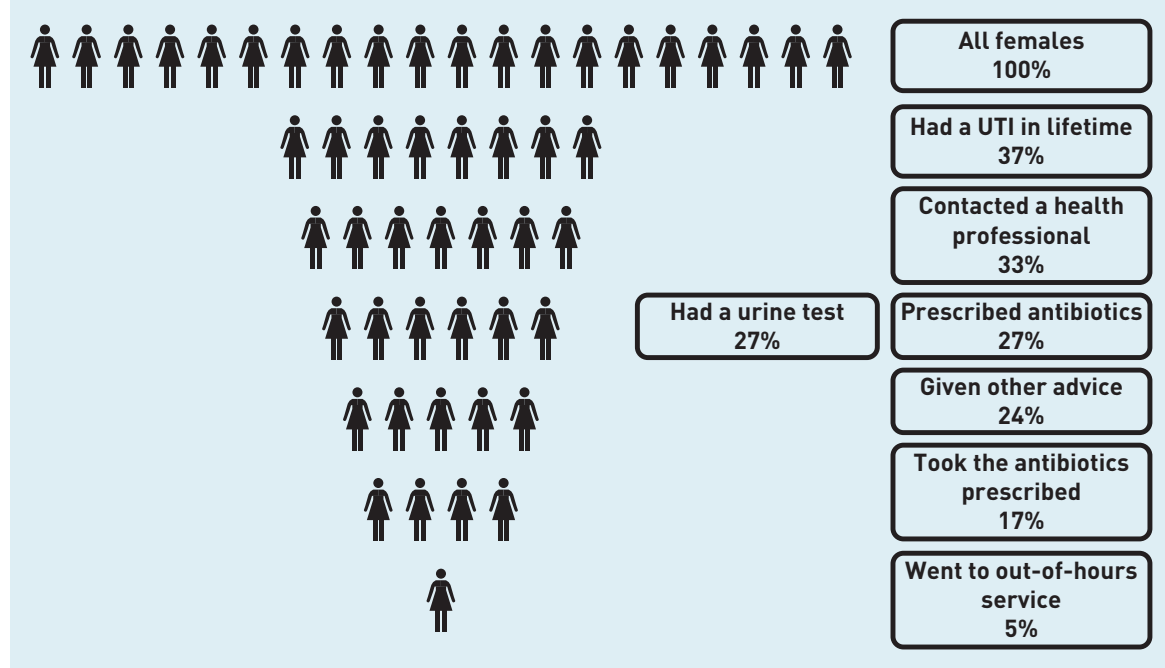

Figure 1. Percentage of females reporting a UTI in their lifetime, and information, advice, and treatment sought with their most recent UTI ( $N=2424)$.
(34\%), and/or persistent symptoms (28\%) were the main reasons for visiting a healthcare professional about UTI. Twothirds $(65 \%, 95 \% \mathrm{Cl}=61.7 \%$ to $68.3 \%)$ contacted their local GP practice, 13\% $(95 \% \mathrm{Cl}=10.8 \%$ to $15.2 \%)$ a pharmacist $5 \%(95 \% \mathrm{Cl}=3.6 \%$ to $6.4 \%)$ an NHS walk-in centre, $4 \%(95 \% \mathrm{Cl}=1.7 \%$ to $6.3 \%)$ an outof-hours GP service, and $4 \% 195 \% \mathrm{Cl}=2.6 \%$ to $5.4 \%$ ) an accident and emergency department. Only $1 \% \quad 195 \% \mathrm{Cl}=0.3 \%$ to $1.7 \%)$ contacted an online doctor. Overall, $14 \%(95 \% \mathrm{Cl}=11.7 \%$ to $16.3 \%)$ contacted an out-of-hours service; only $4 \%$ of these said it was because they could not get an appointment with their GP. Of those aged 16-34 years, 23\% reported contacting an out-of-hours service, whereas $7 \%$ of those aged $\geq 55$ years did so.

\section{Management}

The majority $(76 \% ; 95 \% \mathrm{Cl}=73.2 \%$ to $78.8 \%$ ) of females who contacted a healthcare professional had their urine tested, and just over half of these (52\%) were given the result while they waited, suggesting that this was a point of care dipstick test. In total, $25 \%$ reported that a urine sample was sent for laboratory analysis and 24\% reported telephoning or returning to the surgery for the result.

Of those who contacted a health professional about their last UTI, 74\% (95\% $\mathrm{Cl}=71.1 \%$ to $76.9 \%$ ) reported being prescribed an antibiotic. Those reporting a greater impact on their daily lives $183 \%$ reported that it affected it a great deal versus $65 \%$ who said it didn't affect their daily life) and females aged $\geq 65$ years were more likely to be prescribed antibiotics $184 \%>64$ years versus $73 \%$ of those aged 16-24 years). In total, $88 \%$ were prescribed antibiotics face to face, $5 \%$ over the phone, $4 \%$ delayed or deferred, and $2 \%$ used standby antibiotics. Of those prescribed antibiotics for their most recent UTI, 63\% reported taking them.

Over half of females who had ever had a UTI $(62 \%, 95 \% \mathrm{Cl}=59.1 \%$ to $64.9 \%)$ took non-antibiotic treatment for their most recent UTI: 35\% took extra fluids, 27\% cranberry juice, $21 \%$ used pain-relieving medication such as paracetamol or ibuprofen, and $17 \%$ used cystitis sachets for their most recent UTI. Younger females laged 16-24 years versus those aged 25+ years) were more likely to take cranberry juice (37\%) and to look up information about UTI on the internet (9\%).

Of those who contacted a health professional about their most recent UTI, about three-fifths $159 \%, 95 \% \mathrm{Cl}=55.7 \%$ to $62.3 \%$ ) recalled being given advice or information about their UTI, either about their symptoms (29\%), pain relief $(24 \%)$, or about other treatment they could take $(14 \%)$.

\section{DISCUSSION}

In this population-based face-to-face survey in England, it was found that $37 \%$ of females surveyed reported a UTI at some point in their lifetime, and 28\% reported more than one UTI episode. In total, 11\% reported one, and $6 \%$ more than one UTI in the past year. The vast majority consulted a health professional about their most recent UTI, most often a GP, and almost threequarters of these had a urine test and were prescribed antibiotics, but only twothirds of these females said they actually took the antibiotics as prescribed. Most consultations were in general practice during usual consulting hours, but many younger patients visited the out-of-hours setting.

\section{Strengths and weaknesses}

This face-to-face survey was conducted in communities in the UK and consulted females about their experiences of symptoms attributable to UTIs. The sample is broadly representative of the UK population in terms of age and social class distribution. Some UTIs had occurred a long time previous to the interview and so recall may not have been accurate. Questions were answered by the respondent selecting responses on a computer and this may have minimised social desirability bias. It may be difficult to compare responses with other surveys as the exact wording and order of the questions, as well as the mode of the survey, will influence respondents. 


\section{Comparison with existing literature}

Some previous estimates of incidence of uncomplicated UTI have relied on personal communication and non-peer reviewable analyses. ${ }^{11}$ Citing review articles rather than source articles for incidence figures is common. ${ }^{5}$ The 1997 National Ambulatory Medical Care survey in the US estimated that there are 7 million office visits resulting in a prescription for UTI each year. ${ }^{12}$ Estimates increase to 11.3 million when telephone contacts, self-diagnosis, and use of previously issued prescriptions are taken into account. ${ }^{13}$

A random digit dialling survey of 2000 females in the US asked, 'Has a healthcare professional such as a physician or nurse practitioner ever told you that you had a urinary tract infection? For example, a bladder infection, cystitis, kidney infection, or pyelonephritis?' Females who answered yes to this question and who indicated that a prescription was issued for this were considered to have had a UTI.

The survey found a lifetime risk of UTI of $60.4 \%$ in females, and that $10.8 \%$ of females aged $\geq 18$ years reported at least one presumed UTI in the last 12 months. This was higher in younger females $117.5 \%$ of females aged between 18 and 24 years). Most of these cases were in females who had had two or more previous UTIs. ${ }^{13}$ The authors extrapolated from their survey results to estimate that 11.3 million females in the US were treated with antibiotics for UTI in 1995 at a cost of $\$ 1.6$ billion. ${ }^{13}$

In this current study, females were asked if they had ever had a UTI in order to estimate the overall incidence, including of self-managed episodes, and then asked about help seeking and management. This current study found an estimate of lifetime incidence lower than the US study mentioned above, which is surprising, as the study did not require there to have been interaction with a health professional or the issue of a prescription for a symptomatic episode to be considered a UTI. The estimate therefore would have included some episodes that were selfmanaged, although almost all respondents indicated that they had contacted a health professional for their symptoms. However our estimates of the incidence of UTI in the past year are similar.

A laboratory surveillance study from the Calgary Health Region, Canada, which covers about 1.2 million people, ascertained all laboratory reports positive for community onset UTIs in 2004-2005. A total of 40618 episodes of community onset UTIs were ascertained from 30851 residents, giving an overall annual incidence of 17.5 per 1000. A total of $74 \%$ of the cultures were submitted from ambulatory patients, $18 \%$ from hospital patients within 2 days of admission, and 9\% from nursing home residents. In total, 14\% had more than one UTI during the 2-year surveillance period. ${ }^{14}$ The study covered all age groups and found increasing incidence with age. Sampling thresholds of individual practitioners, which are known to vary widely, would have influenced these figures.? Many females with symptoms of UTI who did not have a sample sent for culture, or who were found to have culture-negative urine, would not have been ascertained using this approach.

A university cohort of 796 sexually active females found an incidence between 0.5 to 0.7 UTIs per year..$^{15} \mathrm{~A}$ prospective study followed 1017 postmenopausal females for 2 years and found an incidence of cultureconfirmed acute cystitis of 0.07 episodes per year. ${ }^{16}$ These are lower than our estimates, and these studies have the advantage of a prospective design, which may diminish risk of recall bias.

A 1989 cross-sectional questionnaire study in a single UK general practice with 661 respondents (response rate 97\%) found a incidence of dysuria over the previous 2 years to be $27 \%$, and the incidence of frequency $34 \%$, while $15 \%$ had received treatment from the practice of urinary symptoms. ${ }^{17}$ Another single UK general practice study sent the Bristol Female Lower Urinary Tract Symptoms (BFLUTS) questionnaire to all 2641 females in the practice, with an $80 \%$ response rate. This study also found that bladder pain and dysuria were commoner among younger females. A Dutch study found that $48 \%$ of females consulting with cystitis reported having more than one episode of UTI in the past year. $^{2}$

Cystitis, one presentation of lower urinary tract infections, was coded in around 1\% of consultations by females in the UK. ${ }^{18}$ Consultation rates for symptoms of urinary tract infection have increased in the Netherlands from 93 to $114 / 1000$ patientyears from 2007 to $2010 .{ }^{2}$

Although females report that use of delayed ('back-up') antibiotics prescribing would be acceptable for UTI, 2,4 few females in this current study reported experience of this strategy being used when they consulted for health care. A questionnaire study of 256 responding females in the Netherlands found that $66 \%$ of females indicated they would be willing to postpone antibiotic use. ${ }^{2}$ A UK trial of different management strategies found that use of 


\section{Funding}

This study was funded internally by Public Health England (Health Protection Agency, Microbiology Services Development Fund 2012/13: No.13).

\section{Ethical approval}

The Ipsos MORI surveys and interviews were undertaken outside the NHS setting and therefore did not need NHS ethical approval. Consent to take part in the survey was by verbal agreement to participate with completion of the questionnaire considered as indicating consent; respondents were not given any financial incentive. Respondents were able to refuse to participate in the questionnaire at any stage in the process. All data were processed in accordance with the Data Protection Act 1998.

\section{Provenance}

Freely submitted; externally peer reviewed.

\section{Competing interests}

Anna Quigley is employed by Ipsos MORI, the company that carried out the survey. Cliodna AM McNulty leads the development and writes evidence-based antibiotic and diagnostic guidance for primary care, including UTI guidance. Any payment for speaking at conferences goes to a research trust fund. Chris C Butler holds publicly funded grants to research aspects of UTI in primary care and has received a fee for lecturing on UTI.

\section{Discuss this article}

Contribute and read comments about this article: bjgp.org/letters delayed prescribing resulted in decreased antibiotic prescribing. ${ }^{19}$

High levels of testing took place, despite evidence for a limited role for dipstick testing in ruling in or ruling out benefit from antibiotic treatment, ${ }^{19}$ and despite recommendations that most cases of uncomplicated UTI can be managed without urine culture. ${ }^{19}$ The Public Health England guidelines provide an evidenced-based algorithm for management of most episodes of UTI symptoms based on symptoms and visual inspection of the urine..$^{20}$

Rates of reported antibiotic prescribing in our study are higher than the 60\% prescribing rate for cystitis found in the study of routine data in the Netherlands. ${ }^{2}$ The low rates of adherence to antibiotics reported here is similar to adherence rates found in a prospective study of antibiotic treatment for lower respiratory tract infection. ${ }^{21}$

\section{Implications for research and practice}

Guidance on appropriate help seeking and additional evidence about self-care for those who do not consult needs further development. The wide gap between reporting being prescribed an antibiotic and taking the antibiotic needs further exploration. Between $34-60 \%$ of those receiving antibiotics for UTI have negative urine cultures. ${ }^{22,23}$ We need to better understand benefits and harms in those who did and did not take antibiotics. If antibiotics were indicated in all of those for whom they were prescribed, then better interventions are required to enhance adherence to these prescriptions. Given that many episodes of symptoms attributable to a UTI resolve without taking antibiotics, a better understanding of the aetiology of these symptoms is required: predictors of low chance of benefit from antibiotic treatment need to be clarified, which in turn would inform appropriate help seeking and management in primary care. Urine tests and antibiotic prescriptions could be reduced if empirical antibiotics were based on symptoms, or back-up prescriptions given in milder cases. The reasons for use of out-of-hours services needs to be explored. 


\section{REFERENCES}

1. Malterud K, Baerheim A. Peeing barbed wire. Symptom experiences in women with lower urinary tract infection. Scand J Prim Health Care 1999; 17(1): 49-53.

2. Willems CS, van den Broek D'Obrenan J, Numans ME, et al. Cystitis: antibiotic prescribing, consultation, attitudes and opinions. Fam Pract 2014; 31(2): 149-155

3. Little P. Merriman R, Turner S, et al. Presentation, pattern, and natural course of severe symptoms, and role of antibiotics and antibiotic resistance among patients presenting with suspected uncomplicated urinary tract infection in primary care: observational study. BMJ 2010; DOI: 10.1136/bmj.b5633.

4. Leydon GM, Turner S, Smith H, Little P, UTIS team. Women's views about management and cause of urinary tract infection: qualitative interview study. BMJ 2010; DOI: 10.1136/bmj.c279.

5. Foxman B. Epidemiology of urinary tract infections: incidence, morbidity, and economic costs. Am J Med 2002; 113 Suppl 1A: 5S-13S.

6. Rosenberg M. Pharmacoeconomics of treating uncomplicated urinary tract infections. Int J Antimicrob Agents 1999; 11(3-4): 247-251; discussion 261-264.

7. Gupta K, Scholes D, Stamm WE. Increasing prevalence of antimicrobial resistance among uropathogens causing acute uncomplicated cystitis in women. JAMA 1999; 281(8): 736-738.

8. Berg AO. Variations among family physicians' management strategies for lower urinary tract infection in women: a report from the Washington Family Physicians Collaborative Research Network. J Am Board Fam Med 1991; 4(5): 327-330.

9. Hillier S, Bell J, Heginbothom M, et al. When do general practitioners request urine specimens for microbiology analysis? The applicability of antibiotic resistance surveillance based on routinely collected data. J Antimicrob Chemother 2006; 58(6): 1303-1306.

10. Platt FW, Keating KN. Differences in physician and patient perceptions of uncomplicated UTI symptom severity: understanding the communication gap. Int J Clin Pract 2007; 61(2): 303-308

11. Kunin CM. Urinary tract infections in females. Clin Infect Dis 1994; 18(1): 1-10; quiz 11-12.
12. Schappert SM. Ambulatory care visits of physician offices, hospital outpatient departments, and emergency departments: United States, 1995. Vital Health Stat 131997; 129: 1-38.

13. Foxman B, Barlow R, D'Arcy H, et al. Urinary tract infection: self-reported incidence and associated costs. Ann Epidemiol 2000; 10(8): 509-515.

14. Laupland KB, Ross T, Pitout JD, et al. Community-onset urinary tract infections: a population-based assessment. Infection 2007; 35(3): 150-153.

15. Hooton TM, Scholes D, Hughes JP, et al. A prospective study of risk factors for symptomatic urinary tract infection in young women. N Engl J Med 1996; 335(7): 468-474.

16. Jackson SL, Boyko EJ, Scholes D, et al. Predictors of urinary tract infection after menopause: a prospective study. Am J Med 2004; 117(12): 903-911.

17. Jolleys JV. The reported prevalence of urinary symptoms in women in one rural general practice. Br J Gen Pract 1990; 40(337): 335-337.

18. Fleming DM, Cross KW, Barley MA. Recent changes in the prevalence of diseases presenting for health care. Br J Gen Pract 2005; 55(517): 589-595.

19. Little P, Moore MV, Turner S, et al. Effectiveness of five different approaches in management of urinary tract infection: randomised controlled trial. BMJ 2010; DOI: 10.1136/bmj.c199

20. British Infection Association, Health Protection Agency. Diagnosis of UTI. Quick reference guide for primary care. https://uww.gov.uk/government/uploads/ system/uploads/attachment_data/file/345784/UTI_quick_ref_guidelines.pdf laccessed 4 Sep 2015).

21. Francis NA, Gillespie D, Nuttall J, et al. Antibiotics for acute cough: an international observational study of patient adherence in primary care. Br J Gen Pract 2012; DOI: 10.3399/bjgp12X649124

22. O'Brien K, Hillier S, Simpson S, et al. An observational study of empirical antibiotics for adult women with uncomplicated UTI in general practice. $J$ Antimicrob Chemother 2007; 59(6): 1200-1203.

23. Little $\mathrm{P}$, Turner $\mathrm{S}$, Rumsby $\mathrm{K}$, et al. Validating the prediction of lower urinary tract infection in primary care: sensitivity and specificity of urinary dipsticks and clinical scores in women. Br J Gen Pract 2010; DOI: 10.3399/bjgp10X514747. 